\title{
Development of a Systematic Diagnostic Strategy of the Repair of a Defective Universal Material Testing Machine (Model SM-100)
}

\author{
B. Kareem and R.K. Apalowo \\ Department of Mechanical Engineering, Federal University of Technology, Akure
}

\begin{abstract}
The main purpose of this research is to carry out breakdown maintenance on a faulty Universal Material Testing Machine (Model SM100) that is hydraulically powered. The machine was first test run to examine its working condition and defects in its operation were noted. The components of the machine were then disassembled and their parts were inspected. The worn defective parts were corrected as appropriate and the machine parts and components were then reassembled. Test specimens were designed and machined for three different types of material and are used to test the performance of the repaired machine. Defects like nonfunctioning load indicator system, broken seals and springs, worn balls on the hydraulic pump valves, inadequate and low viscous hydraulic oil were identified in the machine. The broken seals led to the leakage of oil observed, which in turn reduces the volume of oil inside the pump and the worn balls led to the inadequate supply of oil as well as pressure generated to move the machine columns. Tensile test results on Al and mild steel specimens from the repaired machine were within the standard value. The research work has helped to repair a Universal Material Testing Machine with a little amount of money being expended on the project (monetary savings of about $80 \%$ ) and has also helped in acquiring new practical skills in maintenance engineering. The research work will provides a platform for engineers and laboratory scientists in the institutions, especially in the developing economy, to challenge themselves in maintenance of their equipments and machineries using new low-cost systematic maintenance approach.
\end{abstract}

Keywords: Maintenance, Machine, Tensile Test, Material, Defect, Improved Performance

\section{Introduction}

Maintenance is the combination of all technical and associated administrative actions intended to retain an item in, or restore it to, a state in which it can perform its required function (Chan and Kong, 2005). In his view, Ogunjimi (1996) defined maintenance as the process of putting machines into functional operation to avoid breakdown. Maintenance could also be defined as the act or procedure of keeping or restoring infrastructures such as vehicles, plants and equipment, etc., in good functioning condition or in their original form through regular or periodic checking of repair (Salami, 2003).

Maintenance Engineering is that aspect of engineering that keeps tools and equipments near its new condition as far as possible and retains its design performance (Ademulegun, 2007). Maintenance Engineering has emerged as a specialized discipline in the field of engineering and it incorporates three special areas viz.: design, production and maintenance, it is therefore necessary that at every stage of plant and equipment life, consideration must be given to factors that affect the level of maintenance of machine to achieve maximum utilization and productivity (Randolph, 1956).

Many companies are seeking to gain competitive advantage with respect to cost, quality, service and on-time deliveries. The effect of maintenance on these variables has prompted increased attention to the maintenance area as an integral part of productivity improvement. Maintenance is rapidly evolving into a major contributor to the performance and profitability of manufacturing systems. In fact, some see maintenance as the "last frontier" for manufacturing.

The performance of any facility depends mainly on the level of maintenance effectiveness. That is, if a workshop facility (like the universal testing machine) is abandoned to continue deteriorating, it will be broken down totally at a point in time. Having critically examined this complicated problem, there is need to embark on a proper maintenance analysis of such facility (with universal testing machine, model SM100, as a case study).

This work hence was aimed at examining, diagnosing and maintaining the defective universal material testing machine model SM100 at the material testing laboratory of the Mechanical Engineering department of the Federal University of Technology, Akure (FUTA). The universal testing machine is hydraulically powered and made up of two major units namely; Control Unit and Loading Unit. The hydraulic unit is controlled manually to pump the oil and generate pressure to the moving columns through a connecting hydraulic hose. 


\section{Description of the Machine}

\section{Materials and Methods}

The Universal Material Testing Machine is a hydraulically operated unit providing facilities for tensile and compression tests at loads of up to $100 \mathrm{kN}$ (10tonf). The basic unit requires the hand operated pump. The Machine is supplied with wedge chuck for tensile tests on specimen having circular-section ends, compression platens, a digital load-meter and a dial gauge system for basic deflection measurements.

The basic unit consists of a rigid four-column frame which supports a hydraulic ram. A cross member fitted to the top of the ram carries a loading platform supported on two tie bars. These tie bars are connected at each end by ball joints so that the loading platform is completely self-aligning and loading is purely axial. A strain-gauged load-link is fitted midway up each tie bar and the two links provide an output for the digital load meter which is supported with the equipment.

The space above the loading platform is used for compression test and that below it for tensile tests. Compression platens are supplied for fitting to the top of the loading platform and to the underside of the ram support plate. For tensile tests the specimens are fitted into chuck which screw onto two ball joints; one under the loading platform, the other in the frame base plate. The machine is supported on four sturdy horizontal legs which spread the load and provide adequate stability.

The hydraulic ram has self-sealing coupling and can be connected to the manual pump. The hydraulic ram, manual pump, motorized pump and connecting hoses are all rated for operation up to $200 \mathrm{kN}$ (18 tonf) and therefore have a good margin of safety when operating over the load range of the machine, which is of maximum capacity of $100 \mathrm{kN}$ or 10 tonf (Source: TecQuipment).

\section{Diagnosing of Faults}

The first fault noticed in the defective machine is the non-functioning load meter. In diagnosing for faults, the machine was first operated to determine some operational faults in the machine. This was done with compression test carried out on the machine using a standard test piece. The following faults were detected:

i. The pressure developed from the hydraulic pump was too low to lift the movable columns of the machine.

ii. Oil leakage was observed at the hydraulic hose port of the hydraulic pump.

iii. The machine movable columns were not moving to initiate the compression of the test piece.

After the faults have been detected, the hydraulic pump was removed from the machine and a functioning hydraulic pump was connected to the machine. This was done in order to determine which part of the machine is faulty- the hydraulic pump, the hose or the basic machine unit.

The functioning hydraulic pump was used to test the machine and it was observed that the flow of oil out of the hose outlet was adequate and that the machine columns moved. Hence it can be inferred that the hose is functioning. That is, the machine faults may have been from the hydraulic pump.

In summary the following are the machine's initial defects before dismantling any of its part:

i. $\quad$ Non-functioning digital load meter

ii. Hydraulic oil leakage from the pump

iii. Low viscosity(weak) hydraulic oil

iv. Faulty hydraulic pump unit

\section{Dismantling and Inspection of The Hydraulic Pump}

The faulty hydraulic pump was dismantled and its parts were examined for damage, wear, breakage etc. the following were the procedure followed

i. $\quad$ The shaft nut was loosened

ii. The hydraulic oil was drained off completely

iii. The hydraulic pump was removed from the machine by loosen the hydraulic hose from it

iv. The nuts on both the inlet and outlet valves were loosened and the valve assembly is removed

v. The valves, valve seats and the balls seating on the valves were all checked for wear

vi. The springs were inspected for breakage

vii. The piston was removed from the hand control lever by unlocking the rod connecting them

viii. The piston was inspected for wear

ix. The oil seals in the cover plates at both ends of the hydraulic pump was inspected for wear and/or breakage

x. The tip of the cylinder pump cover plates were inspected for wear

xi. The inner part of the hydraulic pump wall was inspected for wear or scratch

xii. The nut connecting the hydraulic hose to the hydraulic pump was inspected

xiii. The hydraulic hose was used on another machine to pressure test it

xiv. The dismantled components and parts were washed separately with petrol and brush. The washed parts and components were left to dry 
xv. All parts washed were then re-checked for defects like wear, damage, breakage etc.

The following inspected parts are still in good condition

a. The valves and valve seats are still intact with no wear

b. The piston is still good with no wear

c. The cylinder cover has no bend or wear in its tip

d. The hydraulic pump cylinder wall has no wear in both the inner and outer surfaces.

e. The hydraulic hose is working well under pressure

The following were the defects detected during the dismantling and inspection process:

a) The balls on the valves are completely worn out.

b) The springs are broken.

c) The oil seals at both ends of the hydraulic pump are broken and need replacement.

d) The connecting nut between the hydraulic hose and the hydraulic pump is damaged, with worn internal thread.

e) The hydraulic pump lock nut is deformed.

\section{General Maintenance Work Carried Out}

The following are the analyses of the maintenance work carried out on each of the defects detected:

\section{The non-functioning digital load meter}

The load indicator system consists of the digital load meter and a sensor connected to it. Both the load meter and the sensor are completely damaged.

The following were carried out to incorporate a new load indicator system in the machine:

i. An analogue pressure gauge was acquired. The analogue pressure gauge was chosen to replace the digital pressure gauge so as to eliminate the necessity for the electrical connections required.

ii. $\quad$ A tee-nipple was acquired. This is to aid a link among the hydraulic pump, the hydraulic hose and the pressure gauge by connecting them together through its three ports as shown in figure 1 below.

iii. Two threaded connectors were acquired. This is to aid the connection of the tee-nipple with the hydraulic hose and the hydraulic pump.

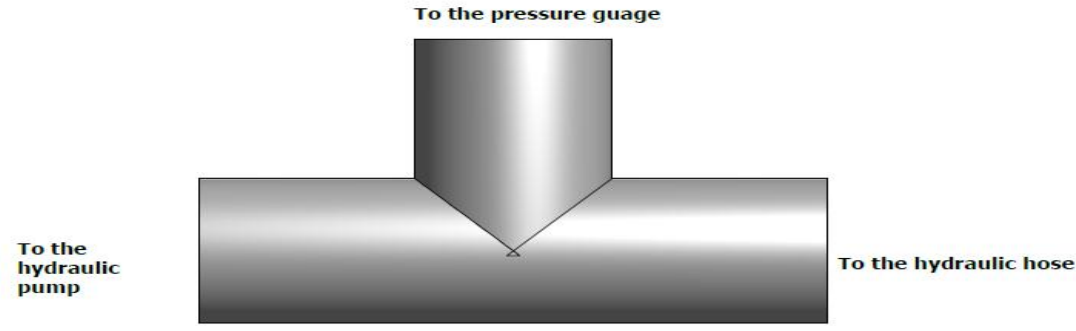

\section{The weak (low viscous) hydraulic oil}

Fig. 1: The Tee-Nipple

The low viscous hydraulic oil was completely drained off before the dismantling process. However, the manufacturer recommended hydraulic was not found around and alternative hydraulic oil recommended by the manufacturer which is SAE 20W50 was used to replace the old hydraulic oil.

\section{Oil leakage and broken seals}

It was apparent that the leakage of oil detected at the hydraulic hose port of the hydraulic pump was due to the broken seals inside the hydraulic pump. The oil seals were replaced with new ones.

\section{The worn balls on the valves}

The balls on the valves are worn and this is responsible for the inadequate supply of oil to the machine reservoir. The balls are replaced.

\section{The broken springs}

The two springs inside the valve housing are broken. This will definitely affect the function of the valve mechanism negatively. Thus, the springs were replaced.

6. The damaged connecting nut between the hydraulic hose and the hydraulic pump 
The nut connecting the hydraulic hose to the hydraulic pump was found to be partially broken and its threads have worn. This will eventually lead to leakage of hydraulic oil when pressure is applied. The nut was replaced by the threaded connector of the tee nipple introduced.

\section{The deformed hydraulic pump lock nut}

The lock nut aids the outflow of oil from the pump as the return of oil back into the hydraulic pump. The deformed lock nut was welded and then ground to achieve its proper shape.

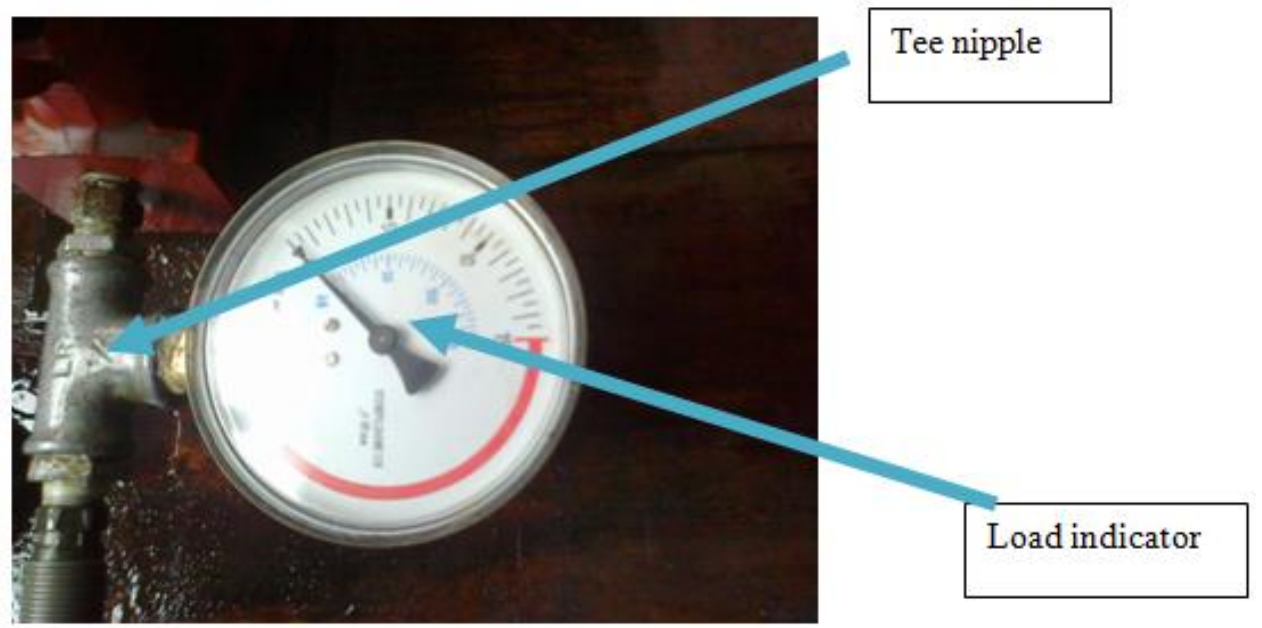

Fig. 2: the new load meter with the Tee Nipple

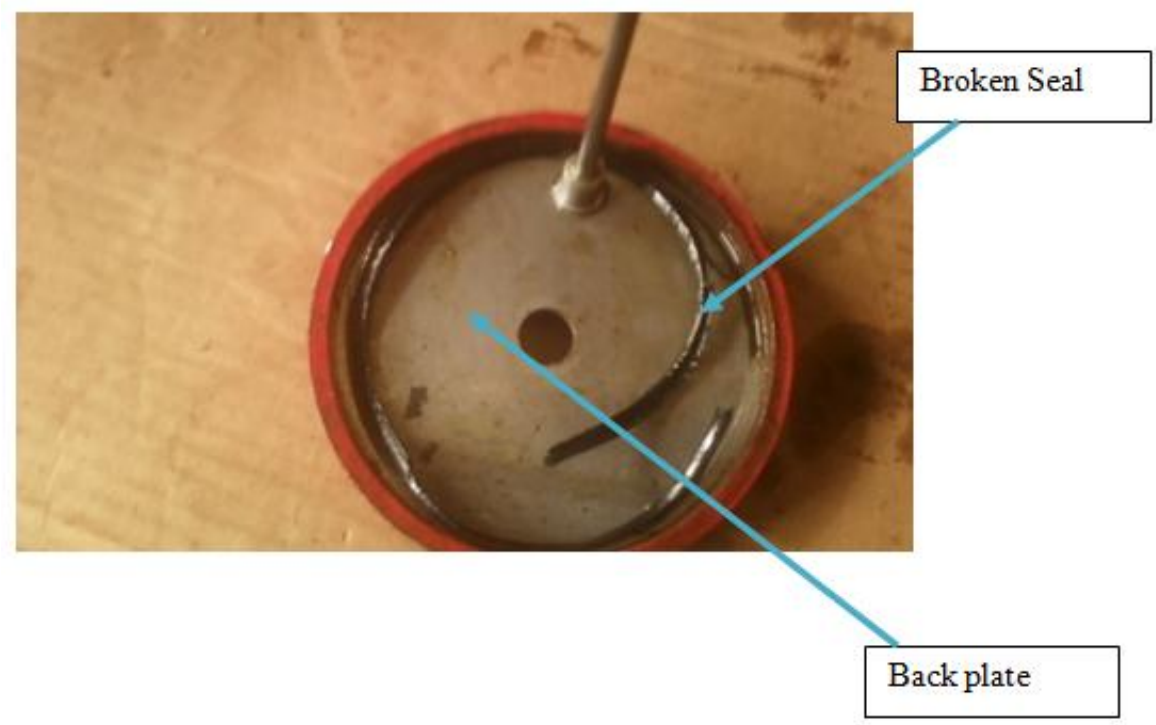

Fig. 3: The cover plate with the broken seal
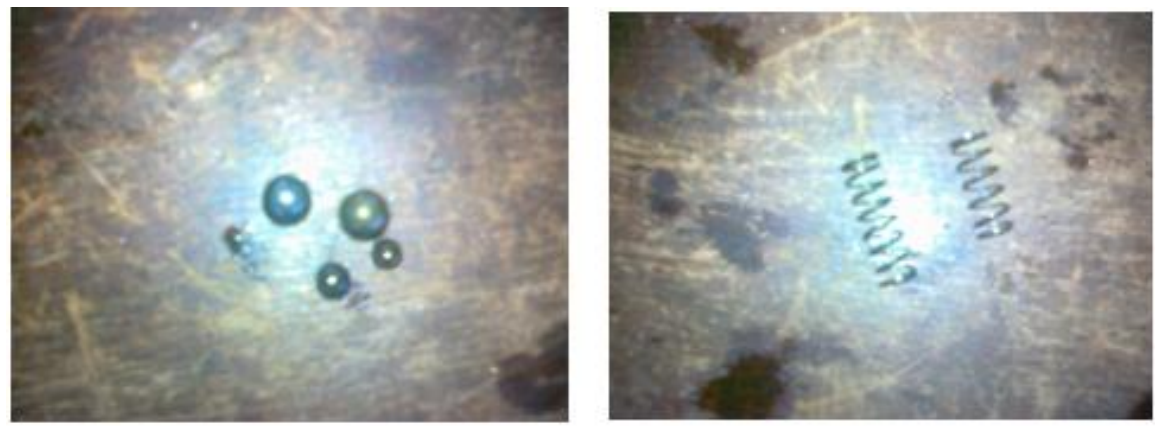

Fig. 4: The worn balls and broken springs 

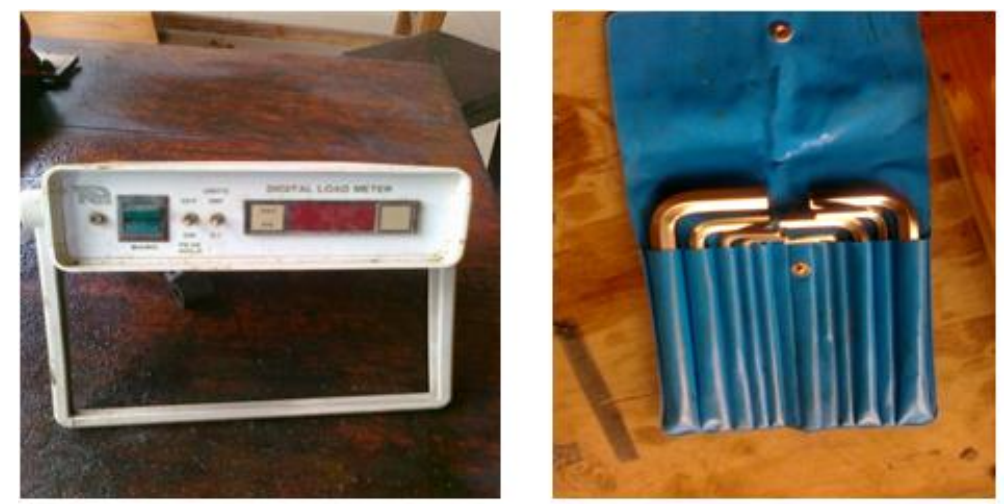

Fig. 5: The damaged load indicator and set of Allen key
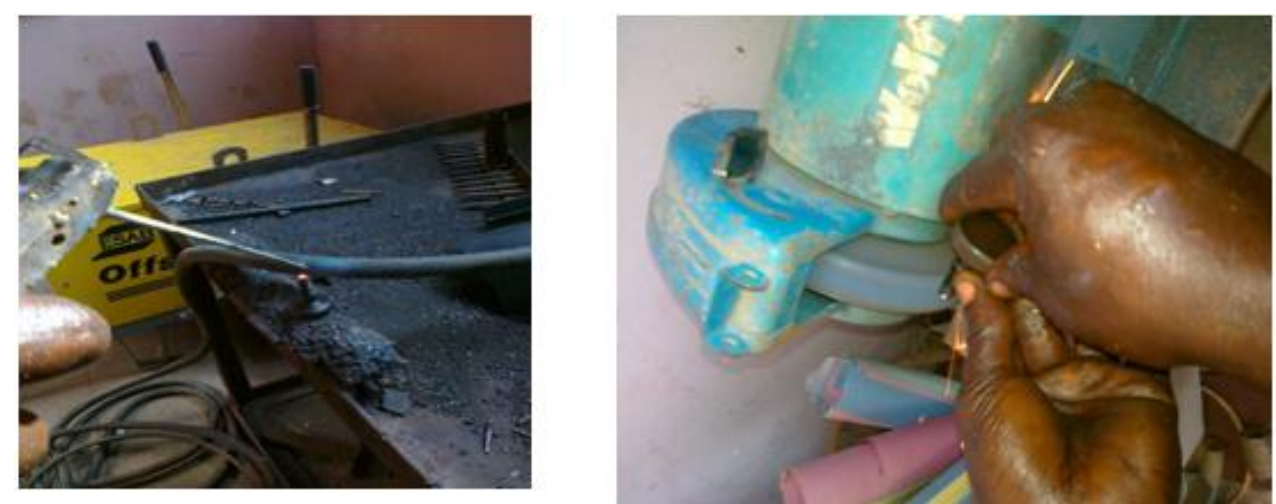

Fig. 6: Welding and grinding of the deformed lock nut tip

\section{Assembly Of The Machine Parts And Components}

The parts and components were assembled as follows:

i. The valve mechanism was first re-coupled by connecting the valve, valve seat and the spring

ii. The valve mechanism was coupled to the valve housing

iii. The housing was the coupled to the cylinder

iv. The cover plate was connected to the cylinder

v. The nuts and bolts were tightened

vi. The hand control lever was connected to the piston head

vii. The tee-nipple was connected to the hydraulic pump through the pump port after the connector has been installed

viii. The pressure gauge was connected to the gauge port of the tee-nipple

ix. The second connector of the tee-nipple was connected to it and the hydraulic hose was connected to the tee-nipple through the connector while the other end of the hose was connected to the machine oil reservoir

x. Hydraulic oil was poured into the hydraulic pump to a recommended level of gauge measured with the gauge on the shaft nut

xi. The shaft nut was then tightened on the hydraulic pump

\section{Testing and Evaluation of the Machine}

\section{Machining of Test Specimen}

The repaired machine was evaluated by tensile tests carried out on it in order to determine working condition of the machine. Two test specimens were machined for each chosen material (Aluminum, mild steel and brass) on the lathe machine to the same size and geometry (i.e., length $100 \mathrm{~mm}$ by step diameters of $30 \mathrm{mmm}, 18 \mathrm{~mm}$ and $10 \mathrm{~mm}$ ). The machine set up and detailed machining operations of the test specimen are shown in figures 7 and 8 respectively. 


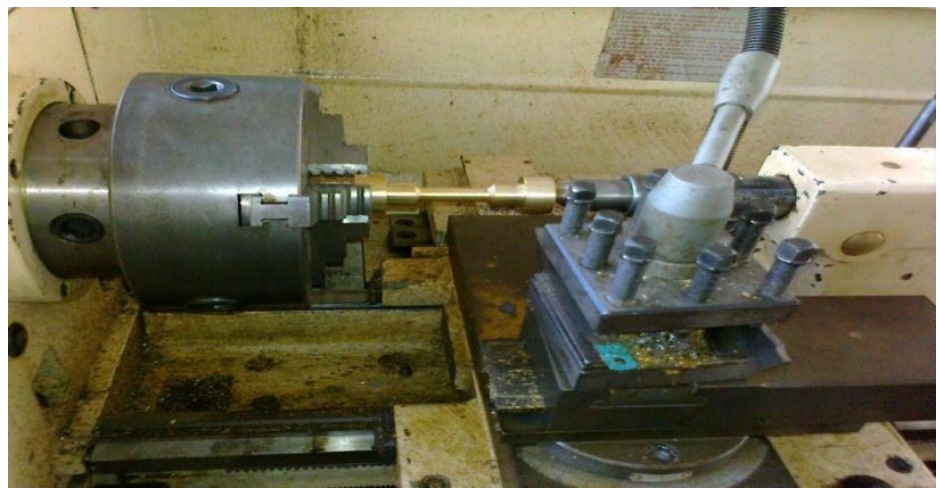

Fig. 9: Machine set-up for machining the Test Specimen

\section{Testing of the machine}

Tensile test was carried out on a specimen of each (one of the two) material using the repaired machine and the test was also done on the other specimens using a well functioning testing machine, as a mean of comparison. The machine set-up is shown in figure 10.
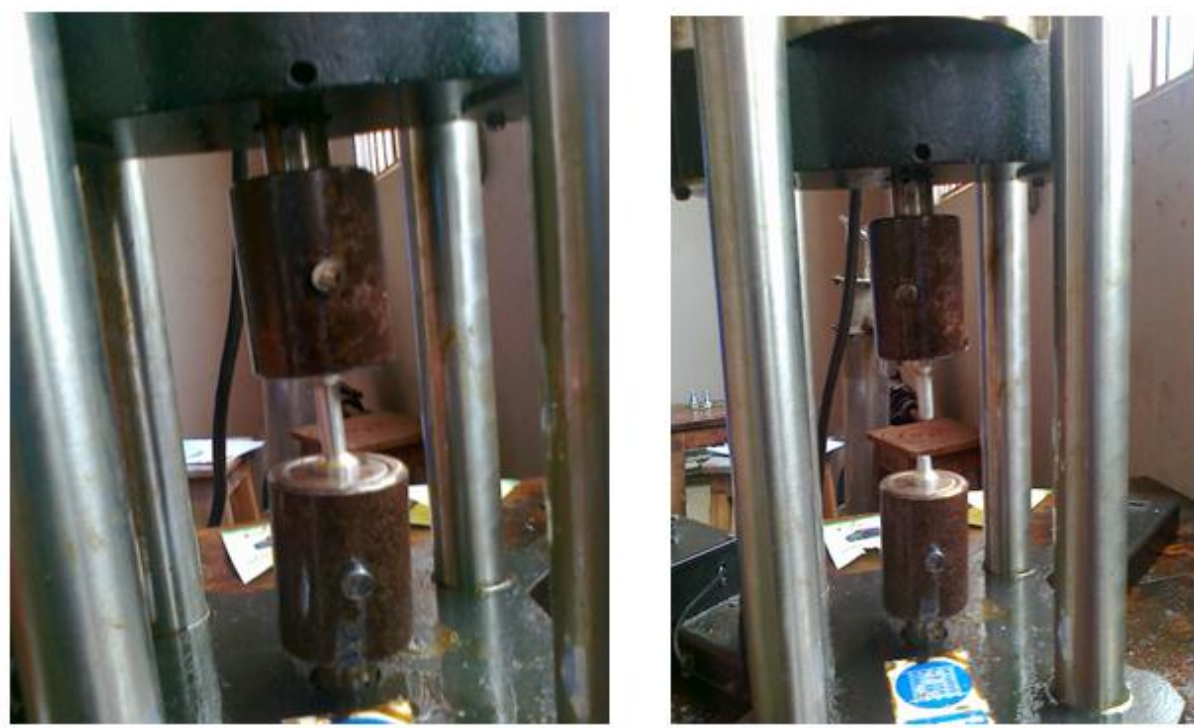

Fig. 10: Machine set-up before and after the experiment

\section{Results and Discussion}

The results obtained for the tensile test carried out using the repaired and the functioning Universal Material Testing Machine are presented in tables 1,2 and 3 as well as figures 11, 12 and 13. The results include the amount of load applied at some various noted extension of the test specimen. As a sign of convention, the data of the repaired machine is represented with subscript 1 while that of the functioning machine is represented with subscript 2 .

Table 1: Force F against extension e obtained for Aluminum

\begin{tabular}{|l|l|l|l|l|l|l|l|l|l|l|}
\hline $\mathbf{F}_{\mathbf{1}}(\mathbf{k N})$ & 0 & 30 & 40 & 43 & 45 & 45 & 43 & 40 & 37 & 32 \\
\hline $\mathbf{F}_{\mathbf{2}}(\mathbf{k N})$ & 0 & 28 & 38 & 41 & 43 & 44 & 41 & 38 & 35 & \\
\hline $\mathbf{e}(\mathbf{m m})$ & 0.0 & 1.0 & 1.5 & 2.0 & 2.5 & 3.0 & 3.5 & 4.0 & 4.5 & 5.0 \\
& & & & & & & & & & \\
\hline
\end{tabular}




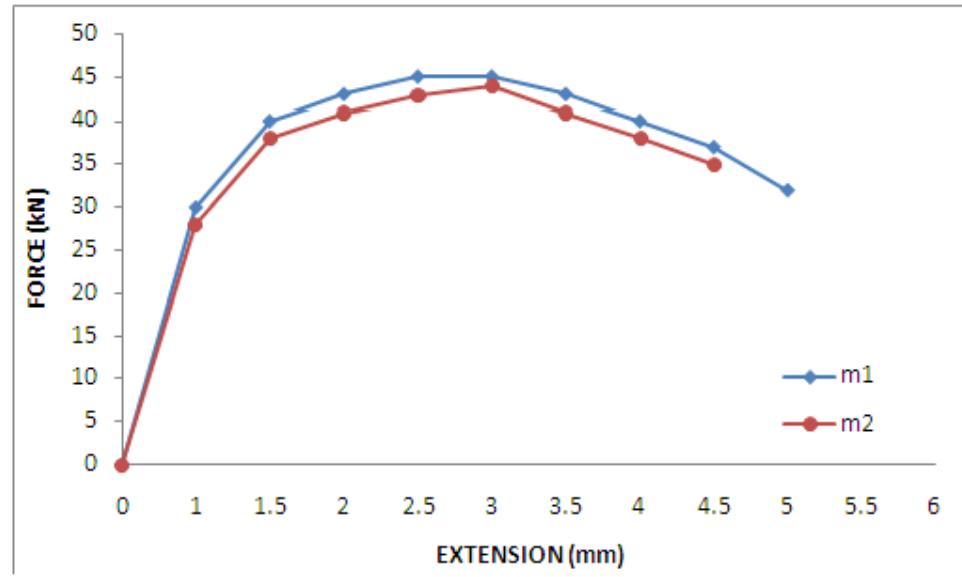

Fig. 11: Graph of Force (kN) against Extension (mm) for Aluminum

Table 2: Force $F$ against extension e obtained for Mild Steel

\begin{tabular}{|l|l|l|l|l|l|l|l|l|l|l|}
\hline $\mathbf{F}_{\mathbf{1}}(\mathbf{k N})$ & 0 & 30 & 35 & 30 & 46 & 49 & 50 & 49 & 47 & 40 \\
\hline $\mathbf{F}_{\mathbf{2}}(\mathbf{k N})$ & 0 & 28 & 32 & 28 & 44 & 46 & 48 & 47 & 44 & 39 \\
\hline $\mathbf{e}(\mathbf{m m})$ & 0.0 & 1.0 & 2.0 & 2.5 & 4.0 & 5.0 & 6.0 & 7.0 & 8.0 & 9.0 \\
\hline
\end{tabular}

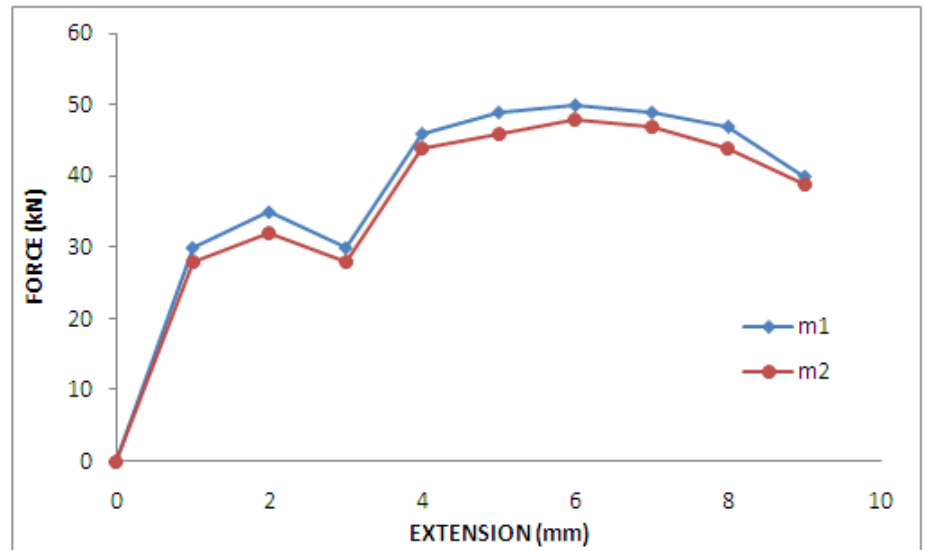

Fig. 12: Graph of Force (kN) against Extension (mm) for Mild steel

Table 3: Force $F$ against extension e obtained for Brass

\begin{tabular}{|l|l|l|l|l|l|l|l|l|l|l|}
\hline $\mathbf{F}_{\mathbf{1}}(\mathbf{k N})$ & 0 & 30 & 35 & 30 & 46 & 49 & 50 & 49 & 47 & 40 \\
\hline $\mathbf{F}_{\mathbf{2}}(\mathbf{k N})$ & 0 & 28 & 32 & 28 & 44 & 46 & 48 & 47 & 44 & 39 \\
\hline $\mathbf{e}(\mathbf{m m})$ & 0.0 & 1.0 & 2.0 & 2.5 & 4.0 & 5.0 & 6.0 & 7.0 & 8.0 & 9.0 \\
\hline
\end{tabular}

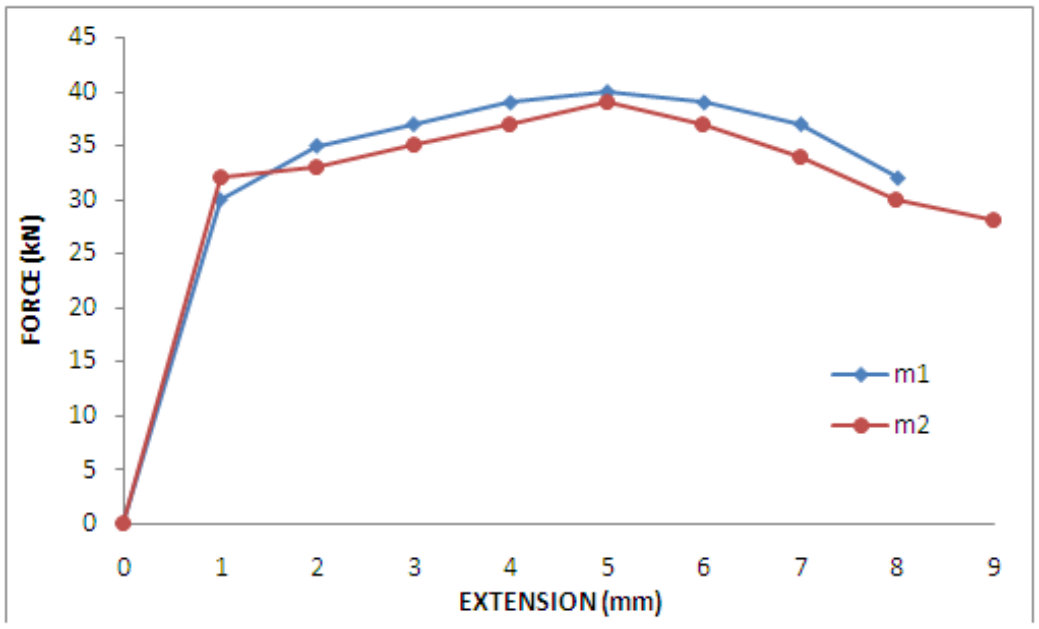

Fig. 13: Graph of Force (kN) against Extension (mm) for Brass 
From the analyses of the results given above, it can be seen that there is no significant difference between the tensile stress-strain curves obtained from using the repaired machine and that obtained using the functioning machine. This outcome is an indication that the repaired machine can perform adequately well as compared with the standard and functioning similar machine.

\section{Conclusion}

This work entailed the maintenance of a defective Universal Material Testing Machine (model SM100) to restore it to its normal working condition. The system load meter was found nonfunctioning and other defects like leakage of oil, broken seals, worn balls, broken springs, broken threaded connector (that connects the hose to the pump), inadequate and low viscous oil in the machine were discovered in the hydraulic pump.

In an attempt to install the new load meter, tee-nipple was acquired and connected to the port of the hydraulic pump with a nut. The tee-nipple connects the load indicator, the hydraulic pump and its hose. The broken seals as well as the worn balls and broken seals were replaced. The old hydraulic oil was drained off and new one was poured into the cylinder. The machine parts were washed and necessary adjustments were made during the re-assembly process.

In respect of this, the leakage of oil was rectified; the discharge of oil from the hydraulic hose was adequate and the machine now works effectively. When the machine was tested, the result obtained was very accurate and within standard value. The same result was gotten in two successive tensile tests for same Aluminum materials having the same geometry and shape as discussed earlier.

In retrospect however, good maintenance practice is an essential function in the overall management of a laboratory facilities like this Universal Material Testing Machine. Without adequate and well planned maintenance programme, the facilities would definitely be faced with continuous problems and interruptions and the purpose for which the facility was meant may not be realized

\section{References}

[1]. Ademulegun, F. (2007). "Engineering Maintenance." Fradem educational Publishers, Ado Ekiti.

[2]. Adesina (2011). Verbal Explanation On The History Of The Defective Universal Testing Machine. FUTA, Akure.

[3]. ASM Handbook (2005): Mechanical Testing and Evaluation, Vol. 8, ASM International, Materials Park.

[4]. Chan, F.T.S. and Kong, S. (2005). "Implementation of Total Productive Maintenance: A Case Study." International Journal of Production Economic.

[5]. Kauppi, P.K. and Yline P. (1995). "Make Maintenance Meaningful" McGraw Hill Publishers, Singapore.

[6]. Ogunjimi, Y.K. (1996). Maintenance Procedure: Industrial Training Workshop Organized by I.T.F. Jos.

[7]. Randolph, W.M. (1956). Industrial Engineering Handbook: Industrial Engineer.

[8]. Salami, K.K. (2003). Engineering Infrastructure: Industrial Training WorkshopOrganized by N.S.E. Ilorin.

[9]. TecQuipment Limited (2014). Maintenance manual for SM100 Universal Material TestingMachine. Nottingham, England 